\section{Histochemical and morphological aspects of fresh frozen bone: a preliminary study}

\author{
F.S. De Ponte, G. Cutroneo, \\ R. Falzea, G. Rizzo, L. Catalfamo, \\ A. Favaloro, G. Vermiglio, M. Runci, \\ A. Centofanti, G. Anastasi \\ Department of Biomedical and Dental \\ Sciences and Morphofunctional Imaging, \\ University of Messina, Italy
}

\section{Abstract}

Bone graft are used in dentistry for the reconstruction of severely atrophic jaws. Fresh frozen bone has no osteogenic property but it has osteoconductive and osteoinductive properties because its matrix contains growth factors such as vascular endothelial growth factor. The purpose of the present study was to evaluate morphological and protein expression characteristics of fresh frozen bone before graft and after six months of graft in patients who needed maxillary reconstruction. After 6 month of graft we observed the presence of viable bone as evidenced by full osteocyte lacunae and by the presence of RANKR, osteocalcin positive cells and vascular endothelial growth factor. In conclusion, our findings show that the fresh frozen bone after six month of graft is for the most part viable bone, encouraging its use as an alternative to autogenous bone for reconstructing maxillary bone defects prior to implant.

\section{Introduction}

In dentistry, bone grafts are widely used for the reconstruction of severely atrophic jaws, caused by trauma, oncologic diseases, oral infections or congenitally missing teeth.

The gold standard for bone grafting is the autogenous bone, harvested from extra- or intra-oral donor sites. ${ }^{1-5}$ Autogenous bone is believed to be the most effective bone graft material and it is still regarded as the "gold standard" for augmentation procedures because of its osteogenic potential, but this graft has limited availability and the surgical harvesting procedures can cause additional morbidity to the patient..$^{6-8}$ By that, it has been therefore focused attention on the use of allogeneic bone graft materials which are all harvested from cadaveric sources, and are successively processed and stored in different ways. ${ }^{9}$

Fresh frozen bone (FFB) has no osteogenic property because of freezing that causes almost total loss of cell viability. Although that, FFB has osteoconductive property and it is potentially osteoinductive because its matrix contains growth factors such as bone morphogenetic protein (BMP) or vascular endothelial growth factor (VEGF); the latter is a key cytokine for angiogenesis, and is also an important regulator of bone remodeling. ${ }^{10-16}$

The absence of universally accepted ridge augmentation success criteria is a significant obstacle in comparing the different studies and surgical techniques using fresh frozen bone, and very often the success of grafting procedures has been measured in terms of implant survival in the areas subjected to bone augmentation. Moreover, few morphological and protein expression study about characteristics of FFB allografts exists and it is believed that histological findings alone did not seem good predictors of the success of grafts over long term. ${ }^{17,18}$ By that the purpose of the present study was to evaluate histological, morphological and molecular characteristics by means of immunofluorescence technique of FFB before grafting (time 0 ) and after six months of graft in patients who needed maxillary reconstruction.

\section{Materials and Methods}

Seven patients received an implant of FFB purchased at the Bank of Cells and Tissue Musculoskeletal of Emilia Romagna, Bologna; FFB has been taken from the tibia in the first $12 \mathrm{~h}$ after the donor's death; it was then disinfected, for at least $72 \mathrm{~h}$ at $-4^{\circ} \mathrm{C}$ in a polychemotherapeutic solution of vancomycin, polymyxin, glazidine, and lincomycin. Finally, bone was irrigated with sterile saline solution, and frozen at $-80^{\circ} \mathrm{C}$. After 6 months patients underwent a second intervention to remove the means of synthesis. Patients signed informed consents and the protocol was approved by local ethics committee.

\section{Histological procedures}

After fixation in $2 \%$ glutaraldehyde and rinses in phosphate buffer $0.13 \mathrm{~mol} / \mathrm{L}, \mathrm{pH} 7.3$, specimens were decalcified in $4.13 \%$ ethylenediaminetetraacetic acid, $\mathrm{pH} 7.2$, for 30 days, dehydrated in ethanol and embedded in paraffin. Five-micrometer-thick sections, obtained in a LEICA microtome, were stained with hematoxylin and eosin (H\&E).

\section{Scanning electron microscopy}

After fixation in $2 \%$ glutaraldheyde, specimens were dehydrated in ethanol and amyl acetate. Then, they were dried at critical-point in a Balzers critical point drier using liquid
Correspondence: Giuseppina Cutroneo, Dipartimento di Scienze Biomediche, Odontoiatriche e delle Immagini Morfologiche e Funzionali, Università di Messina, Policlinico Universitario, Torre Biologica, Via Consolare Valeria 1, 98125 Messina, Italy.

Tel: +39.090 .2213361 - Fax: +39.090 .692449 .

E-mail: gcutroneo@unime.it

Key words: Fresh frozen bone; osteoclast; osteoblast; VEGF; fluorescence antibody technique; histological techniques.

Contributions: GC, FSD, GA, RF, LC, study design; $\mathrm{GV}, \mathrm{AC}, \mathrm{AF}, \mathrm{GR}$, experimental procedures, immunohistochemical processing; MR, LC, RF, collection of biopsies; AF, GV, GR, AC, GC, microscopic analysis, data collection and/or preparation of figures. All authors have reviewed and contributed to the different draft versions of the manuscript and have read and approved the final manuscript.

Conflict of interest: the authors declare no conflict of interest.

Received for publication: 16 March 2016. Accepted for publication: 17 November 2016.

This work is licensed under a Creative Commons Attribution-NonCommercial 4.0 International License (CC BY-NC 4.0).

(C) Copyright F.S. De Ponte et al., 2016

Licensee PAGEPress, Italy

European Journal of Histochemistry 2016; 60:2642 doi:10.4081/ejh.2016.2642

$\mathrm{CO}_{2}$. The fractures surface of bone were mounted on stub and platinum coated with a sputtering system 'Plasma Sciences CrC-100 Turbo Pumped" and observed by Phenom G2 pro scanning electron microscope.

\section{Immunofluorescence}

As per our standard protocol, ${ }^{19,20}$ tenmicrometer-thick section were treated with the following antibodies: rabbit policlonal antiRANK receptor (RANKR) (diluted 1:150; Santa Cruz Biotechnology, Inc., Dallas, TX, USA) to mark osteoclast and mouse monoclonal anti VEGF (diluted 1:50, ABCAM) which were demonstrated with IgG-Texas Red conjugated anti-rabbit (1:100 dilution; Jackson ImmunoResearch Laboratories, West Grove, PA, USA); rabbit policlonal anti-osteocalcin (diluted 1:200; Santa Cruz Biotechnology) to mark osteoblasts which was demonstrated with IgG-FITC conjugated anti-rabbit (1:100 dilution; Jackson ImmunoResearch Laboratories). The negative control was done using only the secondary antibody. Samples were observed with a Zeiss LSM 510 confocal microscope. 

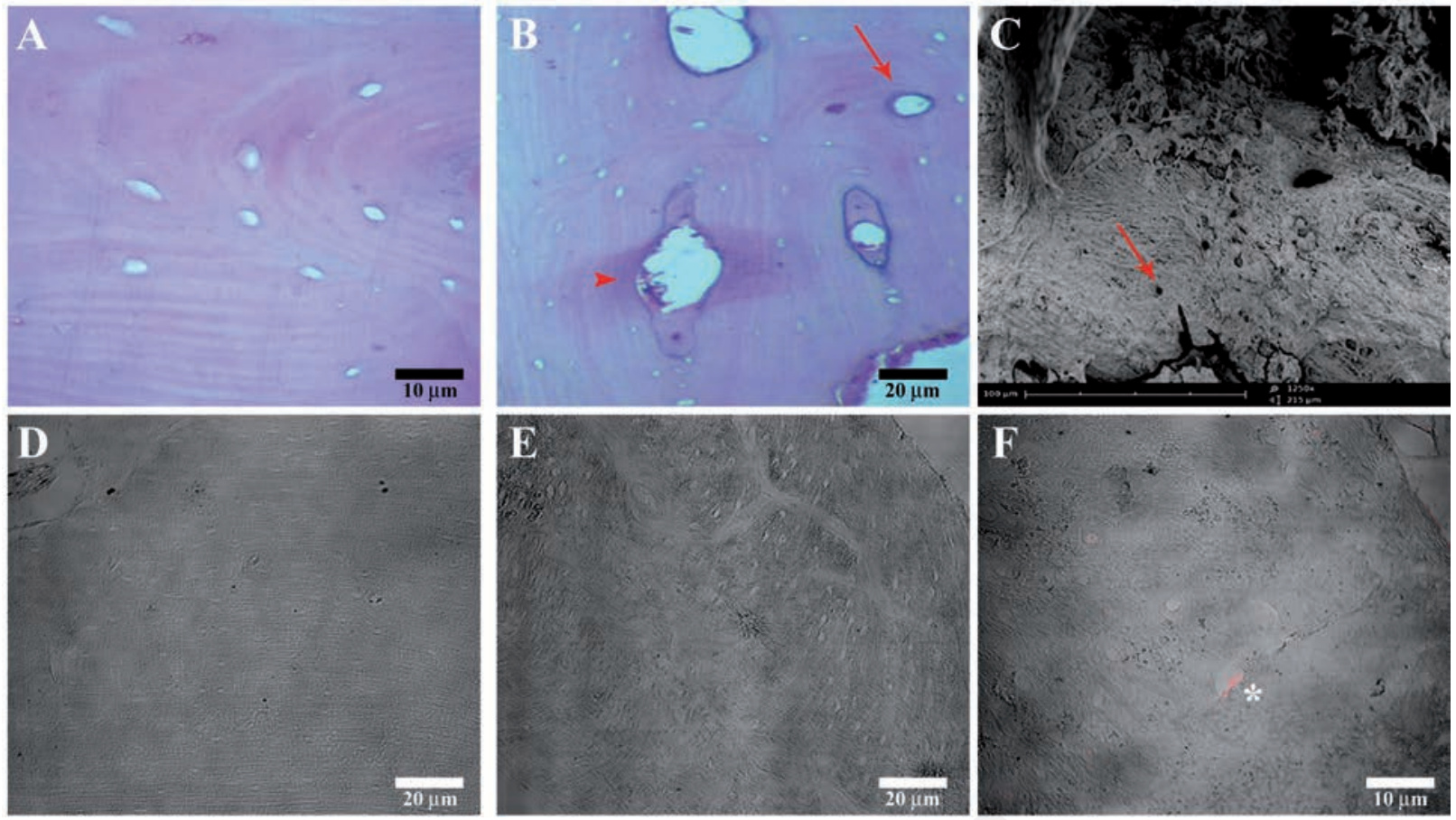

Figure 1. Compound panel of FFB grade 0 images obtained by hematoxylin-eosin, scanning electron microscopy and immunofluorescence techniques. A) Hematoxylin-eosin images show bone tissue characterized by empty osteocyte lacunae and also the presence of empty Volkmann's (head arrow) and Haversian canals (arrow) (B). C) Scanning electron microscopy image shows FFB grade 0 characterized by empty osteocyte lacunae (red arrow) and absence of cellular components. Immunofluorescence reactions images show the absence of fluorescence for RANKR (D) and osteocalcin (E); in F) it is also possible to observe the presence of VEGF fluorescence pattern within some osteocyte lacunae (asterix).
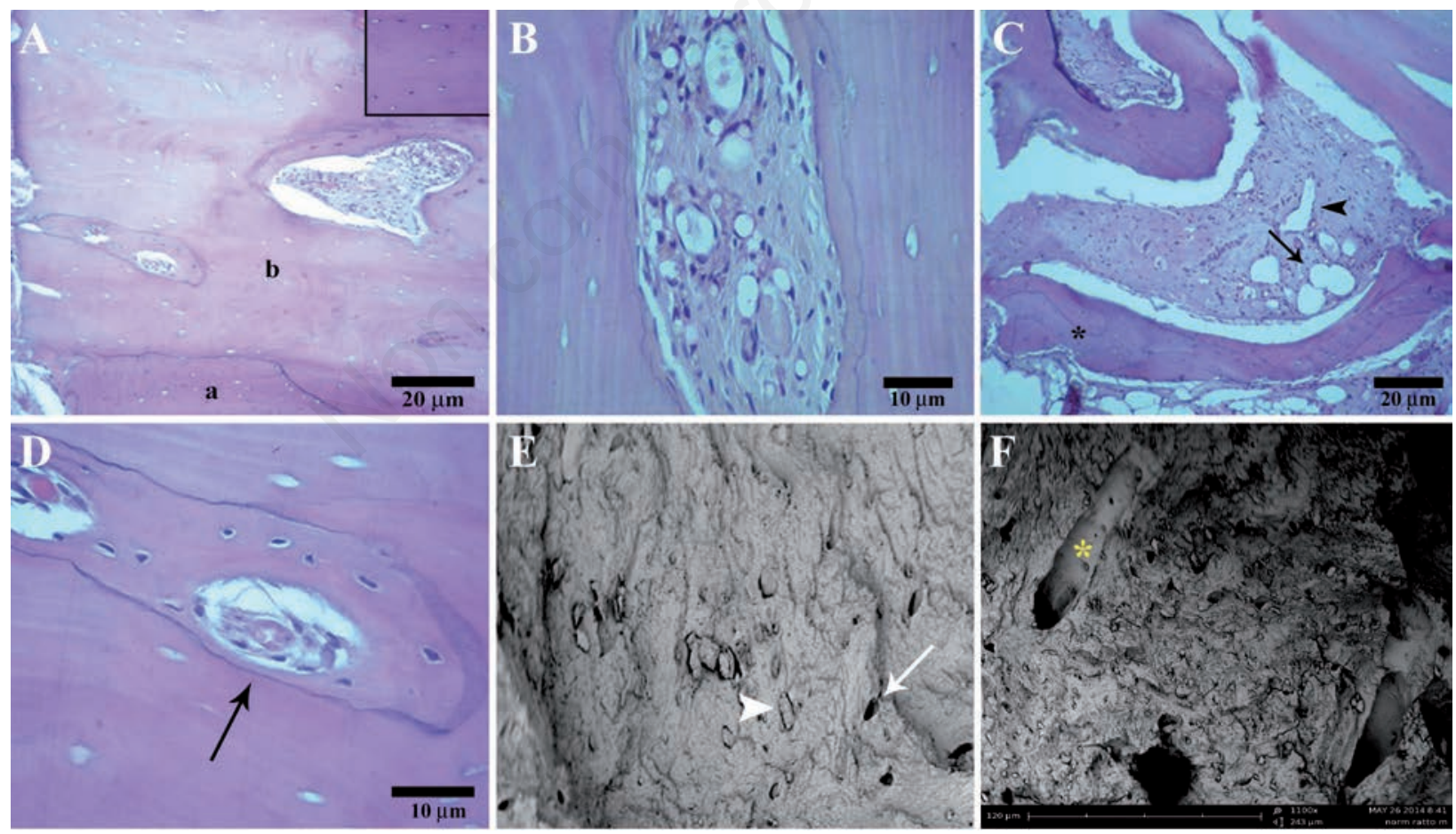

Figure 2. Compound panel of FFB after six month of maxillary graft obtained by hematoxylin-eosin and SEM techniques. A) Image shows the presence of two areas: $a$ and $b$ areas; $a$ areas is characterized by full osteocyte lacunae (magnification in the top right corner). B) Well formed bone marrow in the $b$ area. C) $a$ area with bone lamellae parallel to each other and partially overlapping like roof tiles, alternating to bone lamellae with the same architecture, but with opposite orientation (asterisk) and a well formed bone marrow with adipocyte (arrow) and endothelial cells (head arrow). D) $b$ areas characterized by empty osteocyte lacunae surrounding the bone island (arrow) with full osteocyte lacunae. SEM images show the presence of full (head arrow) and empty (arrow) osteocyte lacunae (E) and Volkmann's canal (F, asterisk). E) magnification: 1500x. 


\section{Results}

\section{Time 0}

H\&E images show that both the cortical and trabecular bone are characterized by empty osteocyte lacunae, empty Volkmann's and Haversian canals and no osteoclasts, osteoblasts and bone marrow have been observed (Figure 1 A,B). Scanning electron microscopy (SEM) shows, in all observed fields the presence of empty osteocyte lacunae and absence of any cellular components (Figure 1C). Immunofluorescence (IF) reactions show the absence of fluorescence for anti-RANKR and anti-osteocalcin antibodies (Figure $1 \mathrm{D}, \mathrm{E}$ ). Slight fluorescence for VEGF within some osteocyte lacunae has been detected (Figure 1F).

\section{Six month}

H\&E images show the presence of two different areas separated by a line: $a$ and $b$ areas (Figure 2A); both in $b$ and $a$ areas it is possible to observe the presence of a well formed bone marrow (Figure $2 \mathrm{~B}, \mathrm{C}$ ) with all its components as adipocytes and endothelial cells. The $a$ area is mainly characterized by full osteocyte lacunae (Figure 2A, top right corner) and by bone lamellae that appear parallel to each other and partially overlapping like roof tiles, alternating to bone lamellae with the same architecture, but with opposite orientation (Figure 2C, asterisk), indicating that bone adsorption and deposition processes occur. The $b$ area is characterized mainly by the presence of empty osteocyte lacunae surrounding some island of bone with full osteocyte lacunae (Figure 2D, arrow).

In the entire sections no evidence of acute or chronic inflammatory infiltrate was observed. SEM results show some empty osteocyte lacunae and wide regions with full osteocyte lacunae (Figure 2E, arrow and head arrow) and full Volkmann's canals (Figure 2F, asterisk). IF results show RANKR positive cells (osteoclasts marker) which are located within lacunae which are probably Howship's lacunae (Figure 3 A,B); images also show aligned osteocalcin positive cells (osteoblasts marker); these cells are located in regions which probably correspond to mineralization site (Figure 3 C,D). VEGF fluorescence pattern has been detected mainly within osteocyte lacunae (Figure 3E).

\section{Discussion}

The aim of this study was to evaluate morphological and protein expression characteristics of FFB at time 0 and after six month of maxillary graft focusing exclusively on morphological aspects. Our H\&E results confirm that the FFB at grade 0 , before graft, is a non viable bone; instead, after 6 month of graft FFB is characterized by two areas, separated by a line, which have different characteristics: $a$ and $b$ areas. The $a$ area is characterized by the presence of a newly formed viable bone as evidenced the presence of cellular elements and blood vessels. Although Literature supports a slower remodeling of corticocancellous blocks because of its limited vascularization, ${ }^{21,22}$ our results show the presence of a well formed vascularization both in the bone marrow and in Volkmann's and Haversian canals.

We also show that the neo-formation of min- eralized bone occurred predominantly adjacent to allogenic bone, which is consistent with the currently available literature; ${ }^{23-29}$ histologically, it is evidenced by the close contact between $a$ and $b$ areas. Works consider the residual bone as necrotic bone; ${ }^{30}$ however, we disagree due to the presence within residual bone, represented by $b$ area, of viable bone island characterized by full osteocyte lacunae and a well formed bone marrow. SEM confirms results obtained by histological techniques, in particular the presence of full osteocyte lacunae and Volkmann's canals after six month of grafts. These data are further supported by IF results which show absence of RANKR and osteocalcin positive cells, which are respectively osteocal-
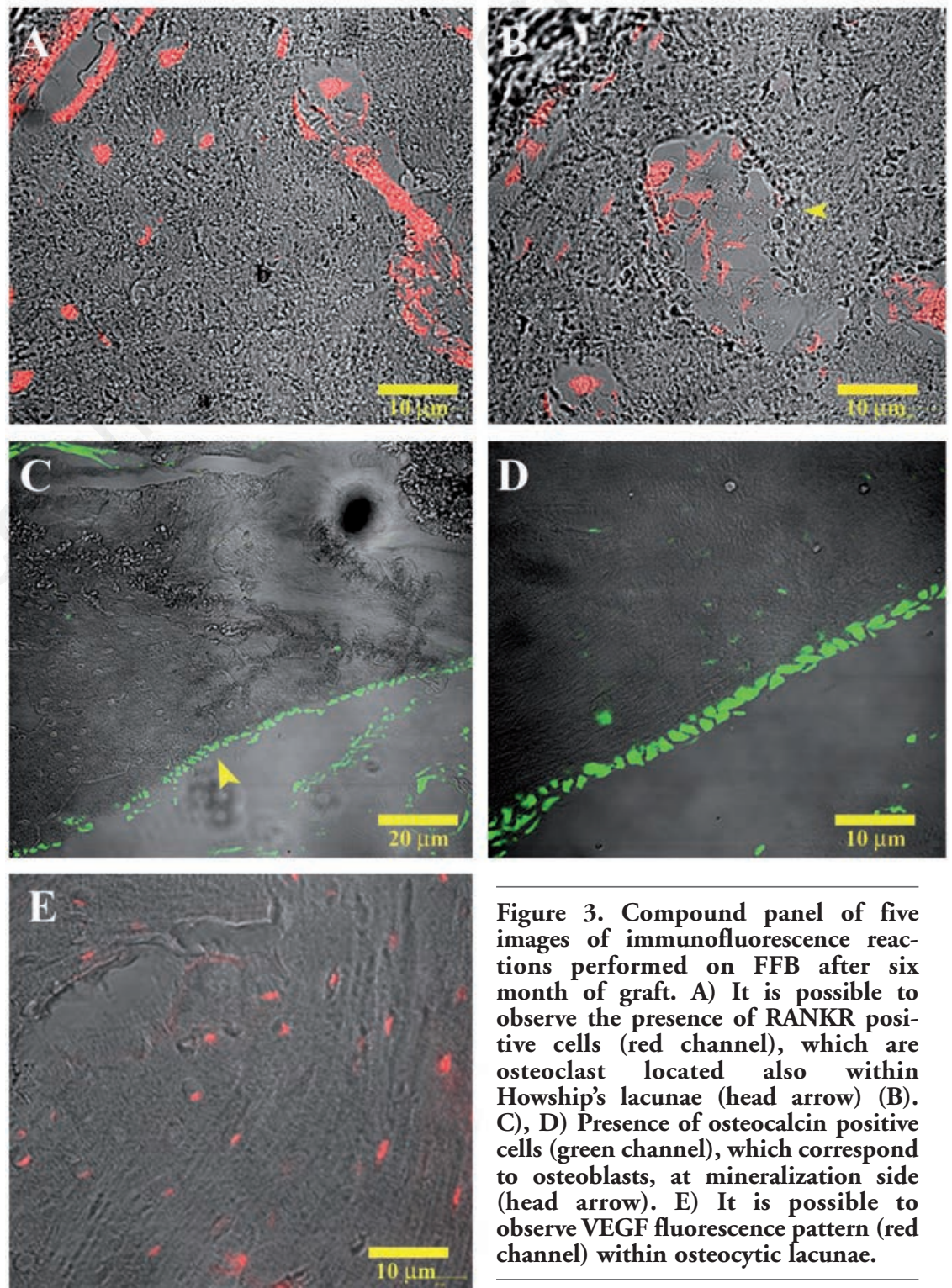

Figure 3. Compound panel of five images of immunofluorescence reactions performed on FFB after six month of graft. A) It is possible to observe the presence of RANKR positive cells (red channel), which are osteoclast located also within Howship's lacunae (head arrow) (B). C), D) Presence of osteocalcin positive cells (green channel), which correspond to osteoblasts, at mineralization side (head arrow). E) It is possible to observe VEGF fluorescence pattern (red channel) within osteocytic lacunae. 
sts and osteoblasts, in grade 0 FFB and the presence of consistent number of RANKR and osteocalcin positive cells in FFB after six month of graft.

In agreement with a previous study, ${ }^{31}$ our IF data show a weakly VEGF fluorescence pattern within osteocyte lacunae in FFB grade 0 and the increase of VEGF fluorescence pattern in FFB after six month of maxillary graft. Several in vitro and in vivo studies, ${ }^{12,14}$ have suggested that VEGF enhances osteoblast differentiation, migration and activity ${ }^{10}$ and osteoclast recruitment. ${ }^{14}$ So it is able to enhance capillary density ${ }^{13}$ playing an important role in angiogenesis associated with osteogenesis. ${ }^{11,12,15,16}$ By that, the presence of VEGF fluorescence pattern in FFB grade 0 supports its osteoinductive properties and the increase in VEGF fluorescence pattern after 6 month of graft could be related to the comparison of osteoblasts, osteoclasts, osteocytes and blood vessels, supporting its role in angiogenesis associated with osteogenesis. All these data suggests that, after bone graft with FFB, two different mechanisms of bone formations could occur at the same time: i) a centripetal mechanism where probably the tight contact between FFB and patients' bone determines and facilitates cell migration, adhesion and proliferation that are necessary for bone regeneration; ii) a centrifugal mechanism towards which cells and growth factors migrate determining the appearance of growth niches which, after 6 month take the form of vital bone island; probably this island long term replace the entire residual nonviable bone. Both these mechanisms could depend on the osteoconductive and osteoinductive properties of fresh frozen bone.

In conclusion, although some reports support that histological findings alone did not seem to be good predictors of the success of grafts $^{17,18}$ we believe that a multi-methodical approach can provide more information on the characteristics of FFB after graft which could be predictive of the success over the long term.

\section{References}

1. Catone GA, Reimer BL, McNeir D, Ray R. Tibial autogenous cancellous bone as an alternative donor site in maxillofacial surgery: a preliminary report. J Oral Maxillofac Surg 1992;50:1258-63.

2. Donovan MG, Dickerson NC, Mitchell JC. Calvarial bone harvest and grafting techniques for maxillary and mandibular implant surgery. Atlas Oral Maxillofac Surg Clin North Am 1994;2:109-22.

3. Misch CM. Maxillary autogenous bone grafting. Oral Maxillofac Surg Clin North Am 2011;23:229-38.
4. Capelli M, Zuffetti F, Del Fabbro M, Testori T. Immediate rehabilitation of the completely edentulous jaw with fixed prostheses supported by either upright or tilted implants: a multicenter clinical study. Int J Oral Maxillofac Implants 2007;22:639-44.

5. Nowzari H, Aalam AA. Mandibular cortical bone graft part 2: surgical technique, applications, and morbidity. Compend Contin Educ Dent 2007;28:274-80.

6. Nkenke E, Radespiel-Tröger M, Wiltfang J, Schultze-Mosgau S, Winkler G, Neukam FW. Morbidity of harvesting of retromolar bone grafts: a prospective study. Clin Oral Implants Res 2002;13:514-21.

7. Cricchio G1, Lundgren S. Donor site morbidity in two different approaches to anterior iliac crest bone harvesting. Clin Implant Dent Relat Res 2003;5:161-9.

8. Acocella A, Bertolai R, Colafranceschi M, Sacco R. Clinical, histological and histomorphometric evaluation of the healing of mandibular ramus bone block grafts for alveolar ridge augmentation before implant placement. J Craniomaxillofac Surg 2010;38:222-30.

9. Gazdag AR, Lane JM, Glaser D, Forster RA. Alternatives to autogenous bone graft: efficacy and indications. J Am Acad Orthop Surg 1995;3:1-8.

10. Wong K. Immediate implantation of endosseous dental implants in the posterior maxilla and anatomic advantages for this region: a case report. Int $\mathrm{J}$ Oral Maxillofac Implants 1996;11:529-33.

11. Dagtekin G, Schiffer R, Klein B, JahnenDechent W, Zwadlo-Klarwasser G. Modulation of angiogenic functions in human macrophages by biomaterials. Biomaterials 2003;24:3395-401.

12. Chen YJ, Wurtz T, Wang CJ, Kuo YR, Yang $\mathrm{KD}$, Huang HC, et al. Recruitment of mesenchymal stem cells and expression of TGF-beta 1 and VEGF in the early stage of shock wave-promoted bone regeneration of segmental defect in rats. J Orthop Res 2004;22:526-34.

13. Geiger D, Debus ES, Ziegler UE, LarenaAvellaneda A, Frosch M, Thiede A, et al. Capillary activity of surgical sutures and suture-dependent bacterial transport: a qualitative study. Surg Infect 200 6:37783.

14. Lalani Z, Wong M, Brey EM, Mikos AG, Duke PJ, Miller MJ, et al. Spatial and temporal localization of FGF-2 and VEGF in healing tooth extraction sockets in a rabbit model. J Oral Maxillofac Surg 2005;63:1500-08.

15. Mori S, Akagi M, Kikuyama A, Yasuda Y, Hamanishi C. Axial shortening during distraction osteogenesis leads to enhanced bone formation in a rabbit model through the HIF-1alpha/vascular endothelial growth factor system. J Orthop Res 2006;24:653-63.

16. Huang H, Ma L, Kyrkanides S. Effects of vascular endothelial growth factor on osteoblasts and osteoclasts. Am J Orthod Dentofacial Orthop 2016;149:366-73.

17. Spin-Neto R, Landazuri Del Barrio RA, Pereira LA, Marcantonio RA, Marcantonio E, et al. Clinical similarities and histological diversity comparing fresh frozen onlay bone blocks allografts and autografts in human maxillary reconstruction. Clin Implant Dent Relat Res 2013;15:4907.

18. Ullmark G, Obrant KJ. Histology of impacted bone-graft incorporation. J Arthroplasty 2002;17:150-7.

19. Anastasi G, Cutroneo G, Rizzo G, Favaloro A. Sarcoglycan sub-complex in normal and pathological human muscle fibers. Eur $\mathrm{J}$ Histochem 2007;51:29-34.

20. Di Mauro D, Gaeta R, Arco A, Milardi D, Lentini S, Runci M, et al. Distribution of costameric proteins in normal human ventricular and atrial cardiac muscle. Folia Histochem Cytobiol 2009;47:605-8.

21. Kahnberg KE, Nystrom E, Bartholdsson L. Combined use of bone grafts and Branemark fixtures in the treatment of severely resorbed maxillae. Int $\mathrm{J}$ Oral Maxillofac Implants 1989;4:297-304.

22. Jensen J, Sindet-Pedersen S, Oliver AJ. Varying treatment strategies for reconstruction of maxillary atrophy with implants: results in 98 patients. J Oral Maxillofac Surg 1994;52:210-6.

23. Acocella A, Bertolai R, Nissan J, Sacco R. Clinical, histological and histomorphometrical study of maxillary sinus augmentation using cortico-cancellous fresh frozen bone chips. J Craniomaxillofac Surg 2011;39:192-9.

24. Spin-Neto R, Stavropoulos A, Coletti FL, Pereira LA, Marcantonio E Jr, Wenzel A. Remodeling of cortical and corticocancellous fresh-frozen allogeneic block bone grafts--a radiographic and histomorphometric comparison to autologous bone grafts. Clin Oral Implants Res 2015;26: 747-52.

25. Proussaefs P, Lozada J. Histologic evaluation of a 9-year-old hydroxyapatite-coated cylindric implant placed in conjunction with a subantral augmentation procedure: a case report. Int $\mathrm{J}$ Oral Maxillofac Implants 2001;16:737-41.

26. Holmquist P, Dasmah A, Sennerby L, Hallman M. A new technique for reconstruction of the atrophied narrow alveolar crest in the maxilla using morselized impacted bone allograft and later placement of dental implants. Clin Implant 
Dent Relat Res 2008;10:86-92.

27. Contar CM, Sarot JR, Bordini J Jr, Galvão GH, Nicolau GV, Machado MA. Maxillary ridge augmentation with fresh- frozen bone allografts. J Oral Maxillofac Surg 2009;67:1280-5.

28. Morelli T, Neiva R, Wang HL. Human histology of allogeneic block grafts for alveolar ridge augmentation: case report. Int $\mathrm{J}$
Periodontics Restorative Dent 2009; 29:649-56.

29. Waasdorp J, Reynolds MA. Allogeneic bone onlay grafts for alveolar ridge augmentation: a systematic review. Int $\mathrm{J}$ Oral Maxillofac Implants 2010;25:525-31.

30. Nissan J, Mardinger 0, Calderon S, Romanos GE, Chaushu G. Cancellous bone block allografts for the augmentation of the anterior atrophic maxilla. Clin Implant Dent Relat Res 2011;13:104-11.

31. Nissan J, Ghelfan 0, Mardinger 0, Calderon S, Chaushu G. Efficacy of cancellous block allograft augmentation prior to implant placement in the posterior atrophic mandible. Clin Implant Dent Relat Res 2011;13:279-85. 